\title{
Renal dysfunction is associated with decline of cognitive function in community-dwelling older adults: Korean frailty and aging cohort study
}

Ji Yoon Kong ${ }^{1 \dagger}$, Jin Sug Kim ${ }^{2 \dagger}$, Min Hye Kang ${ }^{2}$, Hyeon Seok Hwang ${ }^{2}$, Chang Won Won ${ }^{3}$ and Kyung Hwan Jeong ${ }^{2^{*}}$ (D)

\begin{abstract}
Background: Cognitive decline is common in older adults. Similarly, the prevalence of renal dysfunction is also increased in the elderly population. We conducted this study to clarify the relationship between renal dysfunction and decline of cognitive function in community-dwelling elderly population.

Methods: A cross-sectional analysis was performed using data from the Korean Frailty and Aging Cohort Study, a nationwide cohort study. Total 2847 (1333 men, 1514 women) eligible participants were enrolled for this study. The estimated glomerular filtration rate (eGFR, $\mathrm{mL} / \mathrm{min} / 1.73 \mathrm{~m}^{2}$ ) was calculated using the Chronic Kidney Disease Epidemiology Collaboration equation. Global cognitive function was assessed with the Mini-mental State Examination-Korean version. Other domains of cognitive function were tested with the Consortium to Establish a Registry for Alzheimer's disease and the Frontal Assessment Battery.

Results: The mean age of all participants was $76.0 \pm 3.9$ years and eGFR (all in $\mathrm{mL} / \mathrm{min} / 1.73 \mathrm{~m}^{2}$ ) was $77.5 \pm 14.3$. And the mean eGFR was $91.7 \pm 3.2$ in quartile 1,84.9 \pm 1.8 in quartile 2, $76.1 \pm 3.7$ in quartile 3, and $57.2 \pm 10.8$ in quartile 4. In baseline characteristics, participants with lower eGFR tend to have lower cognitive function scores than participant with higher eGFR. In linear regression analysis, eGFR was correlated with the word list memory $(\beta=0.53$, $P=0.005)$, word list recall $(\beta=0.86, P<0.001)$, and word list recognition $(\beta=0.43, P=0.030)$ after adjustment of confounding variables. Moreover, after multivariate adjustment the association with cognitive impairment in quartile 2 was stronger (adjusted OR: 1.535, 95\% Cl: 1.111-2.120, $P=0.009$ ), and the ORs of cognitive impairment were 1.501 (95\% Cl: $1.084-2.079, P=0.014)$ in quartile 3 and 1.423 (95\% Cl: $1.022-1.983, P=0.037$ ) in quartile 4.

Conclusion: In older adults, the immediate, recent memory, and recognition domains were significantly related to renal function. Also, the mild renal dysfunction was independently associated with impairment of global cognitive function. These results suggest that the early stages of renal dysfunction could be an effective target to prevent worsening of cognitive impairment. Therefore, regular monitoring and early detection of mild renal dysfunction in elderly population might be needed.
\end{abstract}

Keywords: Cognitive function, Cohort study, Older adults, Renal function

\footnotetext{
* Correspondence: khjeong@khu.ac.kr

${ }^{\dagger}$ Ji Yoon Kong and Jin Sug Kim contributed equally to this work.

${ }^{2}$ Division of Nephrology, Department of Internal Medicine, College of

Medicine, Kyung Hee University, Seoul, Republic of Korea

Full list of author information is available at the end of the article
}

(c) The Author(s). 2020 Open Access This article is licensed under a Creative Commons Attribution 4.0 International License, which permits use, sharing, adaptation, distribution and reproduction in any medium or format, as long as you give appropriate credit to the original author(s) and the source, provide a link to the Creative Commons licence, and indicate if changes were made. The images or other third party material in this article are included in the article's Creative Commons licence, unless indicated otherwise in a credit line to the material. If material is not included in the article's Creative Commons licence and your intended use is not permitted by statutory regulation or exceeds the permitted use, you will need to obtain permission directly from the copyright holder. To view a copy of this licence, visit http://creativecommons.org/licenses/by/4.0/. The Creative Commons Public Domain Dedication waiver (http://creativecommons.org/publicdomain/zero/1.0/) applies to the data made available in this article, unless otherwise stated in a credit line to the data. 


\section{Background}

Renal dysfunction is common in older adults and strongly related to the risk of cardiovascular and cerebrovascular diseases [1, 2]. Further, the prevalence of cognitive impairment and dementia, which can increase the social and economic burden and reduce the quality of life, is higher in the elderly population [3, 4].

To date, many cross-sectional or longitudinal studies have been performed to determine the relationship between renal function and cognitive function. Patients with chronic kidney disease (CKD) and end-stage renal disease (ESRD) on hemodialysis have a high risk of cognitive impairment $[5,6]$. Moreover, among patients with estimated glomerular filtration rate (eGFR) less than 60 $\mathrm{mL} / \mathrm{min} / 1.73 \mathrm{~m}^{2}$, those with lower eGFR have a higher risk of cognitive impairment [7]. In recent meta-analysis studies, CKD was determined to be an independent somatic risk factor for the development of cognitive decline [8], and cognitive changes were found to occur early in CKD with respect to the orientation-attention and language domains [9]. Moreover, in prior studies of the elderly populations, CKD was found to increase the incidence of cognitive impairment and vascular dementia [10-15]. And cognitive decline was also found to be more rapid in patients with CKD [16].

Most of the previous studies have focused on patients with moderate to severe renal dysfunction including ESRD, whereas few studies have focused on patients with mild renal dysfunction, which takes more than half percentage of all-stage CKD patients [17]. The global prevalence of all-stage CKD, showed differences by literature, was estimated as $8.5-9.8 \%$ and 697.5 million cases were reported worldwide [18-20]. Moreover, the mild renal dysfunction in CKD stage 1 and 2, was accounted for $5.0 \%$. Likewise, in Korea, the estimated prevalence of all-stage CKD was $8.2 \%$, and about $5.7 \%$ was included in stage 1 and 2 [21]. Further, it remains unclear whether renal function is associated with function of different cognitive domains in the communitydwelling older adult population.

The Korean Frailty and Aging Cohort Study (KFACS) is a multicenter, longitudinal study that began in 2016. The participants were community-dwelling older adults aged 70-84 years who were recruited from urban and rural regions across Korea. Other details of the KFACS have been previously described [22, 23]. Recently, KFAC $\mathrm{S}$ data have demonstrated the relationship between cognitive function and calf circumference, sarcopenia, or calorie intake [24-26].

In this study, we attempted to investigate the relationship between renal dysfunction and cognitive decline, and which cognitive function domains are significantly related to renal function in community-dwelling older adults enrolled in KFACS.

\section{Methods}

\section{Study population and design}

This cross-sectional analysis was based on data from the KFACS. Of the 3014 participants who were enrolled in the KFACS during the first and second years (20162017), those without demographic information or baseline laboratory data $(n=6)$ and those who had cerebrovascular disease or dementia in their medical history $(n=161)$ were excluded. A total of 2847 participants (1333 men and 1514 women) were enrolled as the final analysis sample. All study procedures complied with the ethical guidelines of the 1975 Declaration of Helsinki, as revised in 2000.

The KFACS protocol was approved by the Institutional Review Boards (IRBs) of the Clinical Research Ethics Committee of each center, and the approval number from Kyung Hee University Medical Center, the coordinating center, was 2015-12-103. Written informed consents were obtained from all participants or their legal guardians after providing them with sufficient explanation. This study was exempt from review by the IRB of Kyung Hee University Medical Center (IRB number: 2019-09-047).

\section{Cognitive function test}

To evaluate global cognitive function, we used the Minimental State Examination-Korean version (MMSE), which is a screening tool for cognitive decline. Global cognitive impairment was defined as a score of $>1.5$ standard deviation (SD) below the mean of the age-, sex-, and education level-matched Korean norms on MMSE (Supplementary Table 1) [27]. We also conducted neuropsychological tests included in the Consortium to Establish a Registry for Alzheimer's Disease-Korean version (CERAD) assessment battery [28] and Frontal Assessment Battery (FAB) [29]. The CERAD neuropsychological assessment battery is a comprehensive cognitive function assessment tool and consists of eight tests (verbal fluency, Boston naming test, MMSE, word list memory, constructional praxis, word list recall, word list recognition, and constructional praxis recall). In this study, we used the word list memory, word list recall, and word list recognition tests for the immediate, recent memory, and recognition function domains. Digit span forward and digit span backward were used to test the attention-concentration and working memory domains, and the Trail Making Test (TMT) A was used for assessing the processing speed. Further, the FAB was used to test the executive function domain.

\section{Other variables and definitions}

Baseline variables including age; sex; education level; smoking and alcohol consumption; Geriatric Depression Scale-Korean version (GDS) with 15 items [30]; and 
underlying medical problems such as hypertension, diabetes mellitus, dyslipidemia, and coronary artery disease were investigated. Body mass index (BMI) was calculated as weight divided by the square of height $\left(\mathrm{kg} / \mathrm{m}^{2}\right)$. Appendicular skeletal muscle mass (ASM) was measured using DEXA (Hologic DEXA; Hologic Inc., Bedford, MA, USA, and Lunar; GE Healthcare, Madison, WI, USA). ASM index was calculated as the sum of the lean mass from both arms and legs $(\mathrm{kg})$ divided by the square of height $\left(\mathrm{m}^{2}\right)$. Further, eGFR $\left(\mathrm{mL} / \mathrm{min} / 1.73 \mathrm{~m}^{2}\right)$ was calculated using the CKD Epidemiology Collaboration equation [31]. Proteinuria was defined as a score of $\geq 1+$ on a dipstick test in a random urine sample.

\section{Statistical analysis}

Continuous variables are presented as mean \pm SD values, and categorical data are reported as frequencies and percentages. Analysis of variance was used for normally distributed continuous data, the Kruskal-Wallis test was used for non-normally distributed continuous data, with Bonferroni post hoc analysis. Chi-squared test was used for categorical data. Spearman's analyses were used to evaluate the correlation between eGFR and neuropsychological tests. The correlations between zero-score standardized neuropsychological function tests and relevant variables were investigated by linear regression analyses, and sex-stratified analysis was also performed. In multivariate models included significantly associated parameters according to their weight in univariate analysis. Further, the relationship between eGFR and global cognitive impairment was investigated using logistic regression analysis, and the possible confounders including smoking history, alcohol consumption, BMI, GDS, albumin, low-density lipoprotein, hemoglobin, proteinuria, and ASM index were used for adjustment. Logtransformed values of eGFR were used in linear regression, and quartiles of eGFR were used in logistic regression analysis because of a skewed distribution. Unadjusted and adjusted odds ratios (ORs) with 95\% confidence intervals (CIs) were obtained, and a $P$ value of $<0.05$ was considered statistically significant. All statistical analyses were performed using SPSS software version 20.0 (SPSS Inc., Chicago, IL, USA).

\section{Results}

\section{Study population characteristics}

The baseline characteristics of all participants in this study are shown in Table 1 . The mean age was $76.0 \pm 3.9$ years and $1333(46.8 \%)$ were men. The mean eGFR (all in $\mathrm{mL} / \mathrm{min} / 1.73 \mathrm{~m}^{2}$ ) was $77.5 \pm 14.3$ and the mean education duration was $8.4 \pm 5.1$ years. Of the participants, 1637 (57.5\%) had hypertension, $614(21.6 \%)$ had diabetes mellitus, and 222 (8.0\%) had coronary artery disease.
The distribution of participants in each eGFR quartile are also shown in Table 1 . The mean eGFR was $91.7 \pm$ 3.2 in quartile $1,84.9 \pm 1.8$ in quartile $2,76.1 \pm 3.7$ in quartile 3 , and $57.2 \pm 10.8$ in quartile 4 . Participants with lower renal function were older and included more men. They also had higher muscle mass; higher level of triglyceride and high-sensitivity C-reactive protein (hsCRP); lower levels of low-density lipoprotein cholesterol; and higher proportions of patients with hypertension, diabetes mellitus, coronary artery disease, and proteinuria.

The baseline characteristics in terms of cognitive function are shown in Table 2. Generally, participants with lower eGFR had lower cognitive function scores than participant with higher eGFR in MMSE, word list memory, word list recall, and word list recognition. Higher test scores indicate higher cognitive function, except for TMT A in which lower scores are better.

\section{Association between renal function and cognitive function domains}

The association between neuropsychological function tests and eGFR were investigated using Spearman's correlation analyses (Table 3). The result showed positive correlation between eGFR and MMSE, word list memory, word list recall, and word list recognition; and negative correlation between eGFR and TMT A. Linear regression analysis was performed for word list memory, word list recall, and word list recognition (Tables 4, 5 and 6), which showed statistically significant correlation with eGFR and also significant differences between eGFR quartiles (Table 2).

In simple linear regression analysis, word list memory score was positively correlated with $\log$ eGFR $(\beta=1.31$, $P<0.001$ ), education level, alcohol consumption, BMI, albumin, plasma sodium level, and hemoglobin concentration; and negatively correlated with age, male sex, smoking, GDS score, hsCRP, and proteinuria. After multiple linear regression analysis, word list memory score was found to correlate significantly with log eGFR $(\beta=0.53, P=0.005)$ (Table 4).

In word list recall, log eGFR $(\beta=1.47, P<0.001)$, education level, alcohol consumption, BMI, albumin, plasma sodium level, and hemoglobin concentration showed positive correlation; and age, GDS score, hsCRP, and proteinuria showed negative correlation. After adjustment of relevant parameters, the positive correlation between word list recall score and log eGFR $(\beta=0.86, P<0.001)$ remained significant (Table 5).

Table 6 shows the linear regression analyses of word list recognition. In simple linear regression, word list recognition score was positively correlated with log eGFR ( $\beta=0.87, P<0.001$ ), education level, BMI, and hemoglobin concentration; and negatively correlated with age, GDS score, hsCRP, and proteinuria. After 
Table 1 Demographic baseline characteristics of the study group according to renal function

\begin{tabular}{|c|c|c|c|c|c|c|}
\hline & $\begin{array}{l}\text { Overall } \\
(N=2847)\end{array}$ & $\begin{array}{l}\text { Quartile } 1 \\
(n=711)\end{array}$ & $\begin{array}{l}\text { Quartile } 2 \\
(n=712)\end{array}$ & $\begin{array}{l}\text { Quartile } 3 \\
(n=712)\end{array}$ & $\begin{array}{l}\text { Quartile } 4 \\
(n=712)\end{array}$ & $P$ \\
\hline eGFR, mL/min $/ 1.73 \mathrm{~m}^{2}$ & $77.5 \pm 14.3$ & $91.7 \pm 3.2$ & $84.9 \pm 1.8^{a, c}$ & $76.1 \pm 3.7^{\mathrm{a}, \mathrm{b}}$ & $57.2 \pm 10.8^{a, b, c}$ & $<0.001$ \\
\hline \multicolumn{7}{|l|}{ Demographic characteristics } \\
\hline Age, years & $76.0 \pm 3.9$ & $73.7 \pm 3.1$ & $76.2 \pm 3.6^{a, c}$ & $76.7 \pm 4.0^{a, b}$ & $77.4 \pm 3.8^{\mathrm{a}, \mathrm{b}, \mathrm{c}}$ & $<0.001$ \\
\hline Men, n (\%) & $1333(46.8)$ & $262(36.8)$ & $318(44.7)^{\mathrm{a}}$ & $361(50.7)^{\mathrm{a}}$ & $392(55.1)^{a, b}$ & $<0.001$ \\
\hline Education, years & $8.4 \pm 5.1$ & $8.0 \pm 4.8$ & $8.2 \pm 5.0$ & $8.8 \pm 5.1^{\mathrm{a}}$ & $8.7 \pm 5.4^{\mathrm{a}}$ & 0.005 \\
\hline Smoking, n (\%) & $182(6.4)$ & $34(4.8)$ & $52(7.3)$ & $44(6.2)$ & $52(7.3)$ & 0.164 \\
\hline Alcohol consumption, n (\%) & $1416(49.7)$ & $360(50.6)$ & $363(51.0)$ & $364(51.1)$ & $367(51.5)$ & 0.717 \\
\hline \multicolumn{7}{|l|}{ Medical history } \\
\hline Hypertension, n (\%) & $1637(57.5)$ & $335(47.2)$ & $379(53.2)$ & $430(60.4)^{a, b}$ & $493(69.2)^{a, b, c}$ & $<0.001$ \\
\hline Diabetes mellitus, n (\%) & $614(21.6)$ & $135(19.0)$ & $135(19.0)$ & $138(19.4)$ & $206(29.0)^{a, b, c}$ & $<0.001$ \\
\hline Dyslipidemia, n (\%) & $898(31.9)$ & $225(32.1)$ & $218(30.8)$ & $217(31.0)$ & $238(33.7)$ & 0.629 \\
\hline Coronary artery disease, n (\%) & $222(8.0)$ & $35(5.0)$ & $54(7.7)$ & $54(7.7)$ & $79(11.2)^{\mathrm{a}}$ & $<0.001$ \\
\hline \multicolumn{7}{|l|}{ Clinical results } \\
\hline $\mathrm{BMI}, \mathrm{kg} / \mathrm{m}^{2}$ & $24.4 \pm 3.1$ & $24.1 \pm 3.0$ & $24.3 \pm 2.9$ & $24.3 \pm 3.0$ & $24.8 \pm 3.2^{\mathrm{a}, \mathrm{b}}$ & 0.001 \\
\hline ASM index, $\mathrm{kg} / \mathrm{m}^{2}$ & $6.6 \pm 1.1$ & $6.4 \pm 1.1$ & $6.6 \pm 1.1$ & $6.6 \pm 1.2^{\mathrm{a}}$ & $6.7 \pm 1.2^{\mathrm{a}, \mathrm{b}}$ & $<0.001$ \\
\hline $\mathrm{SBP}, \mathrm{mmHg}$ & $131.1 \pm 15.7$ & $130.5 \pm 15.4$ & $131.2 \pm 15.3$ & $131.4 \pm 15.9$ & $131.3 \pm 16.1$ & 0.703 \\
\hline $\mathrm{DBP}, \mathrm{mmHg}$ & $77.4 \pm 9.3$ & $78.0 \pm 9.1$ & $77.4 \pm 9.1$ & $77.5 \pm 9.4$ & $76.8 \pm 9.8$ & 0.137 \\
\hline GDS score & $3.2 \pm 3.7$ & $3.1 \pm 3.6$ & $3.3 \pm 3.7$ & $2.9 \pm 3.5$ & $3.5 \pm 3.8$ & 0.061 \\
\hline \multicolumn{7}{|l|}{ Laboratory results } \\
\hline Albumin, $\mathrm{g} / \mathrm{dL}$ & $4.2 \pm 0.4$ & $4.16 \pm 0.4$ & $4.17 \pm 0.4$ & $4.24 \pm 0.4$ & $4.20 \pm 0.4$ & 0.001 \\
\hline Creatinine, mg/dL & $0.8 \pm 0.3$ & $0.6 \pm 0.1$ & $0.7 \pm 0.1^{\mathrm{a}, \mathrm{c}}$ & $0.9 \pm 0.1^{a, b}$ & $1.2 \pm 0.4^{a, b, c}$ & $<0.001$ \\
\hline Triglyceride, mg/dL & $121.8 \pm 60.9$ & $114.7 \pm 54.1$ & $115.5 \pm 55.5$ & $123.3 \pm 65.8$ & $133.5 \pm 65.7^{a, b, c}$ & $<0.001$ \\
\hline $\mathrm{HDL}-\mathrm{C}, \mathrm{mg} / \mathrm{dL}$ & $52.4 \pm 13.9$ & $54.4 \pm 14.0$ & $53.4 \pm 13.6$ & $52.3 \pm 13.8^{\mathrm{a}}$ & $49.5 \pm 13.9^{a, b, c}$ & $<0.001$ \\
\hline $\mathrm{LDL}-\mathrm{C}, \mathrm{mg} / \mathrm{dL}$ & $108.5 \pm 33.3$ & $111.2 \pm 31.3$ & $109.1 \pm 33.9$ & $108.8 \pm 33.7$ & $104.9 \pm 33.9^{\mathrm{a}}$ & 0.004 \\
\hline Sodium, mmol/L & $141.3 \pm 2.4$ & $141.1 \pm 2.2$ & $141.4 \pm 2.3$ & $141.5 \pm 2.7$ & $141.0 \pm 2.4^{b, c}$ & 0.003 \\
\hline $\mathrm{HbA1c}, \%$ & $6.10 \pm 0.83$ & $6.02 \pm 0.792$ & $6.02 \pm 0.762$ & $6.01 \pm 0.834$ & $6.19 \pm 0.909^{a, b, c}$ & $<0.001$ \\
\hline Hemoglobin, g/dL & $13.4 \pm 1.4$ & $13.4 \pm 1.3$ & $13.5 \pm 1.4$ & $13.6 \pm 1.4$ & $13.2 \pm 1.6^{b, c}$ & $<0.001$ \\
\hline $\mathrm{hsCRP}, \mathrm{mg} / \mathrm{dL}$ & $1.5 \pm 2.6$ & $1.3 \pm 2.2$ & $1.5 \pm 2.8$ & $1.5 \pm 2.5$ & $1.8 \pm 2.9^{a, b, c}$ & $<0.001$ \\
\hline Proteinuria, n (\%) & $84(3.0)$ & $7(8.3)$ & $8(9.5)$ & $17(20.2)$ & $52(61.9)^{a, b, c}$ & $<0.001$ \\
\hline
\end{tabular}

ASM appendicular skeletal muscle mass, BMI body mass index, DBP diastolic blood pressure, eGFR estimated glomerular filtration rate, GDS Geriatric Depression Scale-Korean version, $H b A 1 c$ glycated hemoglobin, $H D L-C$ high-density lipoprotein, $h S C R P$ high-sensitivity C-reactive protein, $L D L-C$ low-density lipoprotein, SBP systolic blood pressure

a $p<0.05$ vs. quartile 1

${ }^{\mathrm{b}} p<0.05$ vs. quartile 2

${ }^{c} p<0.05$ vs. quartile 3

adjustment of confounding variables, the positive correlation between word list recognition score and log eGFR $(\beta=0.43, P=0.030)$ remained significant.

Sex-stratified linear regression analysis of word list memory and word list recall showed significant association with $\log$ eGFR in women. Word list memory was positively correlated with $\log$ eGFR $(\beta=0.75, P=0.005)$ (Supplementary Table 2), and word list recall was also positively correlated with $\log$ eGFR $(\beta=0.87, P=0.002)$ (Supplementary Table 3 ). However, in men, word list memory $(\beta=0.25, P=0.324)$ and word list recall $(\beta=$ $0.51, P=0.051)$ showed positive correlations with eGFR which were not statistically significant (Supplementary Tables 4 and 5). In word list recognition, sex-stratified analysis did not show statistically significant association with $\log$ eGFR in both men and women (table not shown).

\section{Association between renal function and cognitive impairment}

Spearman's correlation analyses showed positive correlation between eGFR and MMSE (Table 3). However, the association between MMSE and eGFR was not linear (Supplementary Table 6). Table 7 shows the association 
Table 2 Cognitive baseline characteristics of the study group according to renal function

\begin{tabular}{|c|c|c|c|c|c|c|}
\hline & $\begin{array}{l}\text { Overall } \\
(N=2847)\end{array}$ & $\begin{array}{l}\text { Quartile } 1 \\
(n=711)\end{array}$ & $\begin{array}{l}\text { Quartile } 2 \\
(n=712)\end{array}$ & $\begin{array}{l}\text { Quartile } 3 \\
(n=712)\end{array}$ & $\begin{array}{l}\text { Quartile } 4 \\
(n=712)\end{array}$ & $P$ \\
\hline MMSE & $25.5 \pm 3.3$ & $25.8 \pm 3.2$ & $25.3 \pm 3.4^{\mathrm{a}}$ & $25.6 \pm 3.3$ & $25.4 \pm 3.5^{\mathrm{a}}$ & 0.013 \\
\hline Word list memory & $16.6 \pm 4.3$ & $17.6 \pm 4.3$ & $16.7 \pm 4.4^{\mathrm{a}}$ & $16.4 \pm 4.2^{\mathrm{a}}$ & $15.9 \pm 4.3^{\mathrm{a}, \mathrm{b}}$ & $<0.001$ \\
\hline Word list recall & $5.5 \pm 2.1$ & $5.9 \pm 2.0$ & $5.6 \pm 2.1^{a}$ & $5.4 \pm 2.1^{a}$ & $5.1 \pm 2.1^{a, b}$ & $<0.001$ \\
\hline Word list recognition & $8.6 \pm 1.9$ & $8.8 \pm 1.8$ & $8.6 \pm 1.9$ & $8.5 \pm 1.9$ & $8.3 \pm 2.0^{\mathrm{a}}$ & $<0.001$ \\
\hline Digit span forward & $6.6 \pm 2.6$ & $6.6 \pm 2.6$ & $6.5 \pm 2.7$ & $6.6 \pm 2.6$ & $6.5 \pm 2.7$ & 0.461 \\
\hline Digit span backward & $4.0 \pm 1.8$ & $4.1 \pm 1.8$ & $3.9 \pm 1.8$ & $4.1 \pm 1.8$ & $4.0 \pm 1.8$ & 0.133 \\
\hline TMT A, sec & $84.3 \pm 63.9$ & $79.9 \pm 57.2$ & $85.4 \pm 63.9$ & $83.8 \pm 67.1$ & $88.1 \pm 66.6$ & 0.060 \\
\hline FAB (range: 0-18) & $13.4 \pm 3.1$ & $13.5 \pm 3.0$ & $13.3 \pm 3.1$ & $13.6 \pm 2.9$ & $13.3 \pm 3.3$ & 0.104 \\
\hline
\end{tabular}

MMSE Mini-mental State Examination-Korean version, TMT A Trail Making Test A, FAB Frontal Assessment Battery

${ }^{a} p<0.05$ vs. quartile 1

${ }^{\mathrm{b}} p<0.05$ vs. quartile 2

${ }^{c} p<0.05$ vs. quartile 3

between eGFR quartiles and global cognitive impairment. In the crude model, the association with global cognitive impairment was found in quartile 2 (unadjusted OR: $1.547,95 \%$ CI: $1.130-2.118, P=0.006)$ than in quartile 1 . The ORs of global cognitive impairment were 1.484 (95\% CI: $1.082-2.035, P=0.014)$ in quartile 3 and 1.465 (95\% CI: $1.067-2.010, P=0.018)$ in quartile 4 compared with quartile 1 . After controlling for potential confounding variables, the association with global cognitive impairment in quartile 2 was stronger (adjusted OR: 1.535, 95\% CI: $1.111-2.120, P=0.009)$. Moreover, the ORs of global cognitive impairment were 1.501 (95\% CI: 1.084-2.079, $P=0.014$ ) in quartile 3 and 1.423 (95\% CI: $1.022-1.983, P=0.037)$ in quartile 4 .

\section{Discussion}

In this cross-sectional study, we showed the association between renal function and neurocognitive function in older adults. The main results were as follows: (1) the immediate, recent memory, and recognition function domains were independently associated with eGFR, (2) particularly in women, the memory domains were significantly associated with eGFR, and (3) mild decline of

Table 3 Univariable Spearman's correlation coefficients

\begin{tabular}{lll}
\hline & \multicolumn{2}{l}{ eGFR, $\mathbf{~ m L} / \mathbf{m i n} / \mathbf{1 . 7 3 m ^ { 2 }}$} \\
\cline { 2 - 3 } & rho & $\boldsymbol{P}$ \\
\hline MMSE & 0.041 & 0.030 \\
Word list memory & 0.141 & $<0.001$ \\
Word list recall & 0.150 & $<0.001$ \\
Word list recognition & 0.092 & $<0.001$ \\
Digit span forward & 0.026 & 0.160 \\
Digit span backward & 0.019 & 0.321 \\
TMT A & -0.040 & 0.034 \\
FAB & 0.011 & 0.564 \\
\hline
\end{tabular}

MMSE Mini-mental State Examination-Korean version, TMT A Trail Making Test A, FAB Frontal Assessment Battery renal function was also strongly associated with global cognitive impairment.

Previous studies have identified the association between renal function and many domains of cognitive function, but they have presented various results. In post-menopausal women, CKD was reported to increase the risk of dysfunction in the global cognition, executive function, language and memory domains [5]. Moreover, in a study in Japanese elderly people, lower levels of eGFR were independently associated with lower cognitive performance in attention and processing speed [10]. In this study, immediate, recent memory, and recognition function domains were associated with renal function in the general elderly population. Further, the immediate and recent memory domains, showed strong association with renal function mainly in women. Among the previous studies that evaluated the association between renal dysfunction and cognitive decline, sex differences were observed in some studies. Mild renal dysfunction has found to be associated with faster cognitive decline in women [32]. Jassal et al. showed albuminuria is associated with greater decline of cognitive function in older adults, particularly in men [33]. However, to our knowledge, the reasons of sex differences in the association between renal dysfunction and cognitive function have not been studied previously. Thus, further investigation might be needed to elucidate the mechanism of sex differences of cognitive decline in patients with renal dysfunction.

Furthermore, these memory domains shared common independent determinants including, eGFR, age, education level, BMI, and GDS. Which means, memory domains can be affected by not only eGFR, but also educational level, emotional and nutritional status, which had been demonstrated before as a risk factor of cognitive dysfunction $[25,28,34,35]$. Soysal et al. showed that the geriatric ESRD patients had lower cognitive function, and it might be affected not only their renal dysfunction, 
Table 4 Simple and multiple linear regression analyses of word list memory score

\begin{tabular}{|c|c|c|c|c|c|c|}
\hline & Simple & & & Multiple & & \\
\hline & estimate & $95 \% \mathrm{Cl}$ & $P$ & estimate & $95 \% \mathrm{Cl}$ & $P$ \\
\hline $\log$ eGFR & 1.31 & $0.94,1.67$ & $<0.001$ & 0.53 & $0.16,0.90$ & 0.005 \\
\hline Age, years & -0.08 & $-0.09,-0.07$ & $<0.001$ & -0.06 & $-0.07,-0.05$ & $<0.001$ \\
\hline Men, $n(\%)$ & -0.10 & $-0.17,-0.02$ & 0.010 & -0.30 & $-0.39,-0.21$ & $<0.001$ \\
\hline Education, years & 0.06 & $0.06,0.07$ & $<0.001$ & 0.06 & $0.05,0.07$ & $<0.001$ \\
\hline Smoking, $n(\%)$ & -0.26 & $-0.41,-0.10$ & 0.001 & -0.07 & $-0.22,0.07$ & 0.334 \\
\hline Alcohol consumption, $n(\%)$ & 0.08 & $0.00,0.15$ & 0.040 & 0.02 & $-0.05,0.09$ & 0.570 \\
\hline $\mathrm{BMI}, \mathrm{kg} / \mathrm{m}^{2}$ & 0.02 & $0.01,0.04$ & $<0.001$ & 0.02 & $0.01,0.03$ & 0.002 \\
\hline GDS score & -0.05 & $-0.06,-0.04$ & $<0.001$ & -0.02 & $-0.03,-0.01$ & $<0.001$ \\
\hline Albumin, $\mathrm{g} / \mathrm{dL}$ & 0.41 & $0.26,0.55$ & $<0.001$ & 0.17 & $0.03,0.30$ & 0.015 \\
\hline $\mathrm{LDL}-\mathrm{C}, \mathrm{mg} / \mathrm{dL}$ & 0.00 & $0.00,0.00$ & 0.939 & NS & . & . \\
\hline Sodium, mmol/L & 0.03 & $0.01,0.04$ & $<0.001$ & 0.01 & $0.00,0.03$ & 0.089 \\
\hline $\mathrm{HbA} 1 \mathrm{c}, \%$ & 0.00 & $-0.05,0.05$ & 0.972 & NS & . & . \\
\hline Hemoglobin, g/dL & 0.04 & $0.02,0.07$ & 0.001 & 0.01 & $-0.02,0.04$ & 0.552 \\
\hline $\mathrm{hsCRP}, \mathrm{mg} / \mathrm{dL}$ & -0.02 & $-0.03,-0.01$ & 0.004 & 0.00 & $-0.01,0.02$ & 0.676 \\
\hline ASM index, $\mathrm{kg} / \mathrm{m}^{2}$ & -0.01 & $-0.04,0.02$ & 0.530 & NS & . & . \\
\hline Proteinuria, $n(\%)$ & -0.28 & $-0.50,-0.07$ & 0.010 & -0.08 & $-0.28,0.12$ & 0.455 \\
\hline
\end{tabular}

ASM appendicular skeletal muscle mass, BMI body mass index, eGFR estimated glomerular filtration rate, GDS Geriatric Depression Scale-Korean version, HbA1C glycated hemoglobin, $h S C R P$ high-sensitivity C-reactive protein, $L D L-C$ low-density lipoprotein cholesterol

Table 5 Simple and multiple linear regression analyses of word list recall scores

\begin{tabular}{|c|c|c|c|c|c|c|}
\hline & \multicolumn{3}{|l|}{ Simple } & \multicolumn{3}{|l|}{ Multiple } \\
\hline & estimate & $95 \% \mathrm{Cl}$ & $P$ & estimate & $95 \% \mathrm{Cl}$ & $P$ \\
\hline log eGFR & 1.47 & $1.11,1.84$ & $<0.001$ & 0.86 & $0.49,1.23$ & $<0.001$ \\
\hline Age, years & -0.08 & $-0.08,-0.07$ & $<0.001$ & -0.06 & $-0.07,-0.05$ & $<0.001$ \\
\hline Men, $n(\%)$ & 0.01 & $-0.07,0.08$ & 0.877 & NS & & \\
\hline Education, years & 0.05 & $0.05,0.06$ & $<0.001$ & 0.04 & $0.04,0.05$ & $<0.001$ \\
\hline Smoking, $n(\%)$ & -0.12 & $-0.28,0.04$ & 0.133 & NS & & \\
\hline Alcohol consumption, $n(\%)$ & 0.16 & $0.08,0.23$ & $<0.001$ & 0.06 & $-0.01,0.13$ & 0.106 \\
\hline $\mathrm{BMl}, \mathrm{kg} / \mathrm{m}^{2}$ & 0.02 & $0.01,0.03$ & $<0.001$ & 0.02 & $0.01,0.03$ & $<0.001$ \\
\hline GDS score & -0.05 & $-0.06,-0.04$ & $<0.001$ & -0.02 & $-0.03,-0.01$ & $<0.001$ \\
\hline Albumin, $\mathrm{g} / \mathrm{dL}$ & 0.33 & $0.19,0.48$ & $<0.001$ & 0.13 & $0.00,0.27$ & 0.057 \\
\hline $\mathrm{LDL}-\mathrm{C}, \mathrm{mg} / \mathrm{dL}$ & 0.00 & $0.00,0.00$ & 0.116 & NS & . & . \\
\hline Sodium, mmol/L & 0.02 & $0.01,0.04$ & 0.008 & 0.01 & $0.00,0.03$ & 0.045 \\
\hline $\mathrm{HbA1c}, \%$ & -0.03 & $-0.08,0.01$ & 0.144 & NS & . & . \\
\hline Hemoglobin, g/dL & 0.06 & $0.03,0.09$ & $<0.001$ & -0.02 & $-0.04,0.01$ & 0.250 \\
\hline hsCRP, mg/dL & -0.02 & $-0.03,0.00$ & 0.021 & 0.00 & $-0.01,0.02$ & 0.664 \\
\hline ASM index, $\mathrm{kg} / \mathrm{m}^{2}$ & 0.02 & $-0.01,0.05$ & 0.201 & NS & & \\
\hline Proteinuria, $n(\%)$ & -0.38 & $-0.59,-0.16$ & $<0.001$ & -0.20 & $-0.40,0.01$ & 0.061 \\
\hline
\end{tabular}

ASM appendicular skeletal muscle mass, BMI body mass index, eGFR estimated glomerular filtration rate, GDS Geriatric Depression Scale-Korean version, HbA1C glycated hemoglobin, $h S C R P$ high-sensitivity C-reactive protein, LDL-C low-density lipoprotein cholesterol 
Table 6 Simple and multiple linear regression analyses of word list recognition scores

\begin{tabular}{|c|c|c|c|c|c|c|}
\hline & Simple & & & Multiple & & \\
\hline & estimate & $95 \% \mathrm{Cl}$ & $P$ & estimate & $95 \% \mathrm{Cl}$ & $P$ \\
\hline log eGFR & 0.87 & $0.51,1.24$ & $<0.001$ & 0.43 & $0.04,0.82$ & 0.030 \\
\hline Age, years & -0.05 & $-0.05,-0.04$ & $<0.001$ & -0.03 & $-0.04,-0.02$ & $<0.001$ \\
\hline Men, $n(\%)$ & 0.02 & $-0.06,0.09$ & 0.635 & NS & & \\
\hline Education, years & 0.03 & $0.02,0.04$ & $<0.001$ & 0.03 & $0.02,0.03$ & $<0.001$ \\
\hline Smoking, $n(\%)$ & -0.11 & $-0.27,0.05$ & 0.169 & NS & & \\
\hline Alcohol consumption, $n(\%)$ & 0.06 & $-0.01,0.14$ & 0.087 & 0.00 & $-0.07,0.08$ & 0.968 \\
\hline $\mathrm{BMI}, \mathrm{kg} / \mathrm{m}^{2}$ & 0.01 & $0.00,0.03$ & 0.022 & 0.01 & $0.00,0.03$ & 0.026 \\
\hline GDS score & -0.03 & $-0.04,-0.02$ & $<0.001$ & -0.01 & $-0.02,-0.00$ & 0.114 \\
\hline Albumin, $\mathrm{g} / \mathrm{dL}$ & 0.12 & $-0.02,0.26$ & 0.105 & NS & & \\
\hline $\mathrm{LDL}-\mathrm{C}, \mathrm{mg} / \mathrm{dL}$ & 0.00 & $0.00,0.00$ & 0.605 & NS & . & . \\
\hline Sodium, mmol/L & 0.01 & $0.00,0.03$ & 0.056 & 0.01 & $0.00,0.03$ & 0.157 \\
\hline $\mathrm{HbA1c}, \%$ & 0.00 & $-0.05,0.04$ & 0.926 & NS & . & . \\
\hline Hemoglobin, g/dL & 0.04 & $0.01,0.07$ & 0.002 & 0.00 & $-0.03,0.03$ & 0.970 \\
\hline hsCRP, mg/dL & -0.02 & $-0.03,0.00$ & 0.013 & -0.01 & $-0.02,0.01$ & 0.326 \\
\hline ASM index, $\mathrm{kg} / \mathrm{m}^{2}$ & 0.02 & $-0.01,0.05$ & 0.230 & NS & . & . \\
\hline Proteinuria, $n(\%)$ & -0.33 & $-0.55,-0.11$ & 0.003 & -0.23 & $-0.45,-0.02$ & 0.035 \\
\hline
\end{tabular}

ASM appendicular skeletal muscle mass, BMI body mass index, eGFR estimated glomerular filtration rate, GDS Geriatric Depression Scale-Korean version, HbA1C glycated hemoglobin, hsCRP high-sensitivity C-reactive protein, $L D L-C$ low-density lipoprotein cholesterol

but also complex geriatric conditions [36]. However, after adjustment of these confounding factors, memory domains showed independent association with eGFR. By comparison with the memory domains, recognition function showed no differences between men and women, and less affected by emotional status.

Moderate to severe renal dysfunction has been recognized as a risk factor of cognitive decline. However, we showed mild decline of renal function was also associated with global cognitive impairment. There might be several mechanisms that explain the association between mild renal dysfunction and global cognitive impairment. One possible explanation is a vascular mechanism including endothelial dysfunction [37-39]. In former studies, minor renal dysfunction has been established as an independent risk factor for cardiovascular disease $[40,41]$. Similar vascular risk factors were reported to affect both the cardiovascular and cerebrovascular systems, and many of them overlapped with risk factors for kidney disease, including smoking, diabetes mellitus, and hypertension [13, 14, 42-44].

This study had several strengths. First, this study was performed with a large cohort comprised of community-dwelling participants nationwide, not clinic-based participants. Thereby, the results of this study could possibly be applied to the general elderly population. Second, cognitive function was assessed using not only MMSE but also CERAD and FAB, thus allowing us to investigate the comprehensive cognitive function. Furthermore, we defined global cognitive impairment as a score of $>1.5$ SD below the mean of MMSE with consideration of age, sex, and education level, which enabled us to more precisely grade the cognitive function of the

Table 7 Logistic regression analysis of the association between estimated glomerular filtration rate quartiles and global cognitive impairment

\begin{tabular}{|c|c|c|c|c|c|}
\hline \multirow[t]{2}{*}{ Variable } & \multirow{2}{*}{$\begin{array}{l}\text { No. of } \\
\text { patients } \\
(\%)\end{array}$} & \multicolumn{2}{|l|}{ Unadjusted } & \multicolumn{2}{|l|}{ Adjusted $^{a}$} \\
\hline & & OR $(95 \% \mathrm{Cl})$ & $P$ & OR $(95 \% \mathrm{Cl})$ & $P$ \\
\hline Quartile 1 & $77(10.8)$ & 1.00 & & 1.00 & \\
\hline Quartile 2 & $113(15.9)$ & $1.547(1.130-2.118)$ & 0.006 & $1.535(1.111-2.120)$ & 0.009 \\
\hline Quartile 3 & $108(15.2)$ & $1.484(1.082-2.035)$ & 0.014 & $1.501(1.084-2.079)$ & 0.014 \\
\hline Quartile 4 & $106(14.9)$ & $1.465(1.067-2.010)$ & 0.018 & $1.423(1.022-1.983)$ & 0.037 \\
\hline
\end{tabular}

${ }^{a}$ Adjusted for smoking, alcohol consumption, body mass index, Geriatric Depression Scale-Korean version score, albumin, low-density lipoprotein cholesterol hemoglobin, proteinuria, and appendicular muscle mass index 
participants [27]. Third, in the elderly population, cognitive function decline is affected not only by aging but also by general medical condition, muscle strength, nutritional status, and emotional status [10, 24-26]. Therefore, it is necessary to determine if the relationship between renal dysfunction and cognitive decline still exists after adjustment for these confounding factors. We considered various laboratory data and medical histories, including proteinuria, muscle mass and emotional status of older adults based on GDS scores, which are known confounding factors.

However, this study also had several limitations. First, we performed only one measurement of serum creatinine and calculated the eGFR, using CKD-EPI equation. Thus, overestimation or underestimation of renal function and misclassification could exist. Also, as the original CKD-EPI equation was developed and validated based on large population composed of Caucasians and African-Americans, and relatively few participants were Asian [31], validation of the equation estimating GFR might be needed. In Korea, the original CKD-EPI equation was valid for Korean population [45]. Moreover, the Korean Society of Nephrology recommends using the original CKD-EPI equations without ethnic adjustment for estimating GFR [46]. But clear conclusion about this ethnic coefficient in Korean may need further investigation. Second, because we used a community-based cohort, the renal function of the participants was not evenly distributed. The proportion of participants with severe renal dysfunction was relatively low, with $12.6 \%(n=360)$ with baseline eGFR $<60 \mathrm{~mL} / \mathrm{min} / 1.73$ $\mathrm{m}^{2}$ and $0.8 \%(n=25)$ with eGFR $<30 \mathrm{~mL} / \mathrm{min} / 1.73 \mathrm{~m}^{2}$ (Supplementary Table 7). This may be explained by the fact that the study sample was composed of volunteers who may have higher cognitive function or may be in better general condition. Thus, we had to divide the study population with eGFR quartiles, not by CKD stages. Also, a potential underestimation of cognitive impairment in this category might be present, and we could not show a dose-dependent relationship between renal dysfunction and global cognitive impairment. Third, this study was crosssectional, which did not allow for us to determine causal associations between renal dysfunction and cognitive impairment. Fourth, in elderly population, CKD increases the chance of taking multiple medications, electrolytes imbalances, and infections. Moreover, it might increase the risk of delirium, which could affect the cognitive function temporarily. However, our data had limited information about delirium of the participants, the effect of delirium on cognitive function in older adults could not be considered. Finally, we used observational data, and even though most of the important confounders were identified and included, unchecked confounders might exist.

\section{Conclusions}

In summary, in the general elderly population, the immediate, recent memory, and recognition domains were significantly related to renal function, and even a mild decline of renal function was independently associated with global cognitive impairment. These results suggest that the early stages of renal dysfunction could be an effective target to prevent worsening of cognitive impairment. Therefore, regular monitoring and early detection of mild renal dysfunction in elderly population might be needed. Further longitudinal studies are needed to ascertain the underlying mechanisms and causal relationships between renal function and cognitive function in older adults.

\section{Supplementary Information}

The online version contains supplementary material available at https://doi. org/10.1186/s12877-020-01862-8.

Additional file 1: Table S1. Cut-points for global cognitive impairment by age-, sex-, education- level matched norms of MMSE-KC. Table S2. Simple and multiple linear regression analyses of word list memory scores in women. Table S3. Simple and multiple linear regression analyses of word list recall scores in women. Table S4. Simple and multiple linear regression analyses of word list memory scores in men. Table S5. Simple and multiple linear regression analyses of word list recall scores in men. Table S6. Simple and multiple linear regression analysis of MMSE. Table S7. Logistic regression analysis of the association between CKD stages and global cognitive impairment.

\section{Abbreviations}

ASM: Appendicular skeletal muscle mass; BMI: Body mass index; CERA D: Consortium to Establish a Registry for Alzheimer's Disease-Korean version; Cl: Confidence interval; CKD: Chronic kidney disease; DBP: Diastolic blood pressure; eGFR: Estimated glomerular filtration rate; ESRD: End-stage renal disease; FAB: Frontal Assessment Battery; GDS: Geriatric Depression ScaleKorean version; HbA1c: Glycated hemoglobin; HDL-C: High-density lipoprotein cholesterol; hsCRP: High-sensitivity C-reactive protein; KFAC S: Korean Frailty and Aging Cohort Study; LDL-C: Low-density lipoprotein cholesterol; MMSE: Mini-mental State Examination-Korean version; OR: Odds ratio; SBP: Systolic blood pressure; TMT A: Trail Making Test A

\section{Acknowledgements \\ The authors are grateful to the Korean Frailty and Aging Cohort Study Group.}

\begin{abstract}
Authors' contributions
JYK and JSK contributed equally to this work. JYK participated in the study design, statistical analysis, writing and revising the manuscript. JSK

participated in the study design and interpretation of data and revising the manuscript. MHK participated in the study design and interpretation of data. $\mathrm{HSH}$ contributed to statistical analysis and revised the manuscript critically. CWW contributed to the data collection process and revised the manuscript critically. KHJ contributed the study design, revised the manuscript critically, and supervised the overall study. All authors read and approved the final manuscript.
\end{abstract}

\section{Funding}

This research was supported by a grant of the Korea Health Technology R \& D Project through the Korean Health Industry Development Institute (KHIDI), funded by the Ministry of Health \& Welfare, Republic of Korea (grant number: 
HI15C3153) and a National Research Foundation of Korea funded by the Ministry of Education (NRF-2017R1A2B4012775). The funding organizations had no role in the design of the study, analysis and interpretation of the data, nor writing the manuscript.

\section{Availability of data and materials}

The datasets used and/or analyzed during the current study are available from the corresponding author on reasonable request.

\section{Ethics approval and consent to participate}

The KFACS protocol was approved by the IRBs of the Clinical Research Ethics Committee of all 10 participating centers (Kyung Hee University, Seoul National University Hospital, Korea University Guro Hospital, Hallym University Medical Center-Chuncheon, Seoul National University Bundang Hospital, Ajou University Hospital, Gyeongsang National University Hospital, Chonnam National University Hospital, and Jeju National University Hospital), including the coordinating center, Kyung Hee University Medical Center, Seoul, Korea (IRB number: 2015-12-103). Written informed consent was obtained from all participants or their legal guardians. This study was exempt from review by the IRB of Kyung Hee University Medical Center (IRB number: 2019-09-047).

\section{Consent for publication}

Not applicable.

\section{Competing interests}

The authors declare that they have no competing interests.

\section{Author details}

'Department of Medicine, Graduate School, Kyung Hee University, Seoul, Republic of Korea. ${ }^{2}$ Division of Nephrology, Department of Internal Medicine, College of Medicine, Kyung Hee University, Seoul, Republic of Korea. ${ }^{3}$ Department of Family Medicine, College of Medicine, Kyung Hee University, Seoul, Republic of Korea.

Received: 5 June 2020 Accepted: 31 October 2020 Published online: 10 November 2020

\section{References}

1. Go AS, Chertow GM, Fan D, McCulloch CE, Hsu CY. Chronic kidney disease and the risks of death, cardiovascular events, and hospitalization. N Engl J Med. 2004:351(13):1296-305.

2. Koren-Morag N, Goldbourt U, Tanne D. Renal dysfunction and risk of ischemic stroke or TIA in patients with cardiovascular disease. Neurology. 2006;67(2):224-8.

3. Salthouse TA. Selective review of cognitive aging. J Int Neuropsychol Soc. 2010;16(5):754-60.

4. Harada CN, Natelson Love MC, Triebel KL. Normal cognitive aging. Clin Geriatr Med. 2013;29(4):737-52.

5. Kurella M, Yaffe K, Shlipak MG, Wenger NK, Chertow GM. Chronic kidney disease and cognitive impairment in menopausal women. Am J Kidney Dis. 2005:45(1):66-76.

6. Murray AM, Tupper DE, Knopman DS, Gilbertson DT, Pederson SL, Li S, Smith GE, Hochhalter AK, Collins AJ, Kane RL. Cognitive impairment in hemodialysis patients is common. Neurology. 2006;67(2):216-23.

7. Kurella Tamura M, Xie D, Yaffe K, Cohen DL, Teal V, Kasner SE, Messe SR, Sehgal AR, Kusek J, DeSalvo KB, et al. Vascular risk factors and cognitive impairment in chronic kidney disease: the chronic renal insufficiency cohort (CRIC) study. Clin J Am Soc Nephrol. 2011;6(2):248-56.

8. Etgen T, Chonchol M, Forstl H, Sander D. Chronic kidney disease and cognitive impairment: a systematic review and meta-analysis. Am J Nephrol. 2012;35(5):474-82

9. Berger I, Wu S, Masson P, Kelly PJ, Duthie FA, Whiteley W, Parker D, Gillespie D, Webster AC. Cognition in chronic kidney disease: a systematic review and meta-analysis. BMC Med. 2016;14(1):206.

10. Lee S, Shimada H, Park H, Makizako H, Lee S, Doi T, Yoshida D, Tsutsumimoto K, Anan Y, Suzuki T. The association between kidney function and cognitive decline in community-dwelling, elderly Japanese people. J Am Med Dir Assoc. 2015;16(4):349 e341-5.

11. Kurella M, Chertow GM, Fried LF, Cummings SR, Harris T, Simonsick E, Satterfield S, Ayonayon H, Yaffe K. Chronic kidney disease and cognitive impairment in the elderly: the health, aging, and body composition study. J Am Soc Nephrol. 2005;16(7):2127-33.

12. Etgen $T$, Sander D, Chonchol M, Briesenick C, Poppert H, Forstl H, Bickel H. Chronic kidney disease is associated with incident cognitive impairment in the elderly: the INVADE study. Nephrol Dial Transplant. 2009;24(10):3144-50.

13. Helmer C, Stengel B, Metzger M, Froissart M, Massy ZA, Tzourio C, Berr C, Dartigues JF. Chronic kidney disease, cognitive decline, and incident dementia: the 3C study. Neurology. 2011;77(23):2043-51.

14. Seliger SL, Siscovick DS, Stehman-Breen CO, Gillen DL, Fitzpatrick A, Bleyer A, Kuller LH. Moderate renal impairment and risk of dementia among older adults: the cardiovascular health cognition study. J Am Soc Nephrol. 2004; 15(7):1904-11.

15. Chen YC, Weng SC, Liu JS, Chuang HL, Hsu CC, Tarng DC. Severe decline of estimated glomerular filtration rate associates with progressive cognitive deterioration in the elderly: a community-based cohort study. Sci Rep. 2017; 7:42690.

16. Buchman AS, Tanne D, Boyle PA, Shah RC, Leurgans SE, Bennett DA. Kidney function is associated with the rate of cognitive decline in the elderly. Neurology. 2009;73(12):920-7.

17. Khatri M, Nickolas T, Moon YP, Paik MC, Rundek T, Elkind MS, Sacco RL, Wright CB. CKD associates with cognitive decline. J Am Soc Nephrol. 2009; 20(11):2427-32.

18. Xie Y, Bowe B, Mokdad AH, Xian H, Yan Y, Li T, Maddukuri G, Tsai CY, Floyd T, Al-Aly Z. Analysis of the global burden of disease study highlights the global, regional, and national trends of chronic kidney disease epidemiology from 1990 to 2016. Kidney Int. 2018;94(3):567-81.

19. Hill NR, Fatoba ST, Oke JL, Hirst JA, O'Callaghan CA, Lasserson DS, Hobbs FD Global prevalence of chronic kidney disease - a systematic review and meta-analysis. PLoS One. 2016;11(7):e0158765.

20. Collaboration GBDCKD. Global, regional, and national burden of chronic kidney disease, 1990-2017: a systematic analysis for the global burden of disease study 2017. Lancet. 2020;395(10225):709-33.

21. Park Jl, Baek H, Jung HH. Prevalence of chronic kidney disease in Korea: the Korean National Health and nutritional examination survey 2011-2013. J Korean Med Sci. 2016;31(6):915-23.

22. Won CW, Lee Y, Choi J, Kim KW, Park Y, Park H, Oh IH, Ga H, Kim YS, Jang $\mathrm{HC}$, et al. Starting construction of frailty cohort for elderly and intervention study. Ann Geriatr Med Res. 2016:20(3):114-7.

23. Won CW, Lee S, Kim J, Chon D, Kim S, Kim CO, Kim MK, Cho B, Choi KM, Roh $\mathrm{E}$, et al. Korean frailty and aging cohort study (KFACS): cohort profile. BMJ Open. 2020;10(4):e035573.

24. Kim S, Kim M, Lee Y, Kim B, Yoon TY, Won CW. Calf circumference as a simple screening marker for diagnosing sarcopenia in older Korean adults: the Korean frailty and aging cohort study (KFACS). J Korean Med Sci. 2018 : 33(20):e151.

25. Park JM, Lee J, Kim Y, Won CW, Kim YJ. Calorie intake and cognitive function in the elderly: data from the Korean frailty and aging cohort study (KFACS). J Nutr Health Aging. 2019;23(10):930-6.

26. Kim M, Won CW. Sarcopenia is associated with cognitive impairment mainly due to slow gait speed: results from the Korean Frailty and Aging Cohort Study (KFACS). Int J Environ Res Public Health. 2019:16(9):1491.

27. Lee DY, Lee KU, Lee JH, Kim KW, Jhoo JH, Youn JC, Kim SY, Woo SI, Woo J. A normative study of the mini-mental state examination in the Korean elderly. J Korean Neuropsychiatr Assoc. 2002;41(9):508-25.

28. Lee DY, Lee KU, Lee JH, Kim KW, Jhoo JH, Kim SY, Yoon JC, Woo SI, Ha J, Woo Jl. A normative study of the CERAD neuropsychological assessment battery in the Korean elderly. J Int Neuropsychol Soc. 2004;10(1):72-81.

29. Kim TH, Huh Y, Choe JY, Jeong JW, Park JH, Lee SB, Lee JJ, Jhoo JH, Lee DY, Woo Jl, et al. Korean version of frontal assessment battery: psychometric properties and normative data. Dement Geriatr Cogn Disord. 2010;29(4):363-70.

30. Bae JN, Cho MJ. Development of the Korean version of the geriatric depression scale and its short form among elderly psychiatric patients. J Psychosom Res. 2004;57(3):297-305.

31. Levey AS, Stevens LA, Schmid CH, Zhang YL, Castro AF 3rd, Feldman HI, Kusek JW, Eggers P, Van Lente F, Greene T, et al. A new equation to estimate glomerular filtration rate. Ann Intern Med. 2009;150(9):604-12.

32. Sajjad I, Grodstein F, Kang JH, Curhan GC, Lin J. Kidney dysfunction and cognitive decline in women. Clin J Am Soc Nephrol. 2012;7(3):437-43.

33. Jassal SK, Kritz-Silverstein D, Barrett-Connor E. A prospective study of albuminuria and cognitive function in older adults: the rancho Bernardo study. Am J Epidemiol. 2010;171(3):277-86. 
34. Godin O, Dufouil C, Ritchie K, Dartigues JF, Tzourio C, Peres K, Artero S, Alperovitch A. Depressive symptoms, major depressive episode and cognition in the elderly: the three-city study. Neuroepidemiology. 2007; 28(2):101-8.

35. Yaffe K, Blackwell T, Gore R, Sands L, Reus V, Browner WS. Depressive symptoms and cognitive decline in nondemented elderly women: a prospective study. Arch Gen Psychiatry. 1999;56(5):425-30.

36. Soysal P, Isik AT, Buyukaydin B, Kazancioglu R. A comparison of end-stage renal disease and Alzheimer's disease in the elderly through a comprehensive geriatric assessment. Int Urol Nephrol. 2014;46(8):1627-32.

37. Ochodnicky P, Vettoretti S, Henning RH, Buikema H, Van Dokkum RP, de Zeeuw D. Endothelial dysfunction in chronic kidney disease: determinant of susceptibility to end-organ damage and therapeutic response. J Nephrol. 2006;19(3):246-58.

38. Annuk M, Zilmer M, Fellstrom B. Endothelium-dependent vasodilation and oxidative stress in chronic renal failure: impact on cardiovascular disease. Kidney Int Suppl. 2003:84:S50-3.

39. Seliger SL, Salimi S, Pierre V, Giffuni J, Katzel L, Parsa A. Microvascular endothelial dysfunction is associated with albuminuria and CKD in older adults. BMC Nephrol. 2016;17(1):82.

40. Pereg D, Tirosh A, Shochat T, Hasdai D, Metabolic L. Nutrition assessment in young adults I: Mild renal dysfunction associated with incident coronary artery disease in young males. Eur Heart J. 2008;29(2):198-203.

41. Pinkau T, Hilgers KF, Veelken R, Mann JF. How does minor renal dysfunction influence cardiovascular risk and the management of cardiovascular disease? J Am Soc Nephrol. 2004;15(3):517-23.

42. Ikram MA, Vernooij MW, Hofman A, Niessen WJ, van der Lugt A, Breteler MM. Kidney function is related to cerebral small vessel disease. Stroke. 2008; 39(1):55-61.

43. Fox CS, Larson MG, Leip EP, Culleton B, Wilson PW, Levy D. Predictors of new-onset kidney disease in a community-based population. JAMA. 2004; 291(7):844-50.

44. Seliger SL, Sarnak MJ. Subclinical vascular disease of the brain in dialysis patients. Am J Kidney Dis. 2007;50(1):8-10.

45. Jeong TD, Lee W, Yun YM, Chun S, Song J, Min WK. Development and validation of the Korean version of CKD-EPI equation to estimate glomerular filtration rate. Clin Biochem. 2016:49(9):713-9.

46. Teo BW, Zhang L, Guh JY, Tang SCW, Jha V, Kang DH, Tanchanco R, Hooi LS, Praditpornsilpa K, Kong X, et al. Glomerular filtration rates in Asians. Adv Chronic Kidney Dis. 2018;25(1):41-8.

\section{Publisher's Note}

Springer Nature remains neutral with regard to jurisdictional claims in published maps and institutional affiliations.

Ready to submit your research? Choose BMC and benefit from:

- fast, convenient online submission

- thorough peer review by experienced researchers in your field

- rapid publication on acceptance

- support for research data, including large and complex data types

- gold Open Access which fosters wider collaboration and increased citations

- maximum visibility for your research: over $100 \mathrm{M}$ website views per year

At $\mathrm{BMC}$, research is always in progress.

Learn more biomedcentral.com/submissions 